PROCEEDINGS OF THE

AMERICAN MATHEMATICAL SOCIETY

Volume 125, Number 1, January 1997, Pages 209-217

S 0002-9939(97)03578-8

\title{
FIXED POINT SUBALGEBRAS OF RATIONAL HIGHER-DIMENSIONAL NON-COMMUTATIVE TORI
}

\author{
CARLA FARSI AND NEIL WATLING
}

(Communicated by Palle E. T. Jorgensen)

\begin{abstract}
We consider the fixed point subalgebras of higher-dimensional rational non-commutative tori induced by finite order automorphisms derived from a diagonal extension of the canonical $S L(2, \mathbb{Z})$ action on rotation algebras. We give an alternative characterization and determine their $K$-theory.
\end{abstract}

\section{INTRODUCTION}

Non-commutative tori are probably the most well understood examples of noncommutative, not necessarily type I, $C^{*}$-algebras but even then several questions still remained unanswered. Firstly we would like to recall the definition of a noncommutative $d$-dimensional torus, $\mathcal{A}_{\Theta}[8]$. Let $\Theta \in \operatorname{Hom}\left(\mathbb{Z}^{d} \wedge \mathbb{Z}^{d}, \mathbb{T}\right)$ be a character of $\mathbb{Z}^{d} \wedge \mathbb{Z}^{d}$. Then $\mathcal{A}_{\Theta}$ is the universal unital $C^{*}$-algebra generated by $d$ unitary operators $u_{1}, \ldots, u_{d}$ subject to the relations $u_{j} u_{i}=\Theta\left(e_{i} \wedge e_{j}\right) u_{i} u_{j}, i, j=1, \ldots, d$, where $\left\{e_{1}, \ldots, e_{d}\right\}$ is a standard basis for $\mathbb{Z}^{d}$. Note for $d=2$ we shall denote $\mathcal{A}_{\Theta}$ by $A_{\theta}$ with $\theta \in[0,1)$ since the algebra in this case is determined by a real number $\theta$ and the relation $u_{2} u_{1}=e^{2 \pi i \theta} u_{1} u_{2}$. This algebra is isomorphic to the crossed product $C^{*}$-algebra $C(\mathbb{T}) \rtimes_{\theta} \mathbb{Z}$ for the action of $\mathbb{Z}$ on $C(\mathbb{T})$ induced by the rotation by $2 \pi \theta$ on the circle $\mathbb{T}$ and consequently is also referred to as a rotation algebra. In this note we are interested in automorphisms of higher-dimensional non-commutative tori and the study of the corresponding fixed point subalgebras.

For the 2-dimensional non-commutative torus or rotation algebra, $A_{\theta}$, the situation has been considered in some depth $[3,4,5,6,9,10,11,12,13,14,15,16,17]$. The automorphism group of irrational rotation algebras (up to inner automorphisms) has recently been described by Elliott and Rørdam [9] as generated by the automorphisms induced by $S L(2, \mathbb{Z})$ and an automorphism inducing a determinant -1 transformation on $K_{1}\left(A_{\theta}\right) \cong \mathbb{Z}^{2}$ (of which there is no explicit description).

In particular the natural action $\pi$ of $S L(2, \mathbb{Z})$ on the rotation algebra $A_{\theta}$, first described in $[6,17]$, is given by $\pi_{A}\left(u_{1}\right)=e^{\pi i a c \theta} u_{1}^{a} u_{2}^{c}, \pi_{A}\left(u_{2}\right)=e^{\pi i b d \theta} u_{1}^{b} u_{2}^{d}$ where $A=\left(\begin{array}{ll}a & b \\ c & d\end{array}\right) \in S L(2, \mathbb{Z})$. Considering the fixed point algebras arising from this action there are four interesting cases [10]. These are determined by the automorphisms coming from the four non-trivial finite subgroups of $S L(2, \mathbb{Z})$, namely, up

Received by the editors January 27, 1995 and, in revised form, July 27, 1995.

1991 Mathematics Subject Classification. Primary 46L80, 46L40.

Key words and phrases. Non-commutative tori, fixed point $C^{*}$-algebras, classification.

(C)1997 American Mathematical Society 
to conjugacy, $\mathbb{Z}_{2}, \mathbb{Z}_{3}, \mathbb{Z}_{4}$ and $\mathbb{Z}_{6}$. The generators of these cyclic groups $\mathbb{Z}_{2}, \mathbb{Z}_{3}, \mathbb{Z}_{4}$ and $\mathbb{Z}_{6}$ can be taken to be the following elements of $S L(2, \mathbb{Z})$ respectively,

$$
\left(\begin{array}{cc}
-1 & 0 \\
0 & -1
\end{array}\right),\left(\begin{array}{cc}
-1 & -1 \\
1 & 0
\end{array}\right),\left(\begin{array}{cc}
0 & 1 \\
-1 & 0
\end{array}\right) \text { and }\left(\begin{array}{cc}
0 & -1 \\
1 & 1
\end{array}\right)
$$

It would be very interesting to know the structure and classify the fixed point subalgebras and the crossed products associated to elements of $S L(2, \mathbb{Z})$.

For $\theta$ rational, $\theta=\frac{p}{q}$, there is a complete description of the fixed point subalgebras $A_{\theta}^{\mathbb{Z}_{i}}$ for all four cases $i=2,3,4,6[3,10,11,12,13]$, as trivial matrix bundle $C^{*}$-algebras over the 2 -sphere $S^{2}$ with generic fiber $M_{q}$ and exceptional fibers at four points of $S^{2}$ for $i=2$ and three points of $S^{2}$ for $i=3,4,6$ (at least for $q>i$; for $q \leq i$ an analogous description is true but there are fewer fixed points and their orders could be smaller). In fact a similar description is also valid for the crossed products $A_{\theta} \rtimes \mathbb{Z}_{i}$. For $\theta$ irrational, in the case of the first of these, usually referred to as the flip automorphism, a result of Bratteli and Kishimoto [4] has shown the fixed point subalgebra is in fact an AF algebra, a fact conjectured but as yet unproven for the other three cases.

These results lead naturally to ask what can be done in higher dimensions. Unfortunately the situation here is far from complete with very little known. The fact that the same is true for the automorphism group for higher dimensions is one major reason. However, some progress has been made recently. Since most noncommutative tori have just been shown to be inductive limits of circle algebras [2], the determination of the automorphism group of $A_{\Theta}$ may now be possible. We have already considered the generalized flip automorphism, $\Sigma$, elsewhere [14], in the completely irrational case and recently Boca has shown that the fixed point subalgebra is indeed AF [2], as in dimension two. Here we wish to consider the case of rational higher-dimensional tori where it is possible to say something more about the structure of $A_{\Theta}$. Now $\Theta$ is said to be rational if Range $(\Theta)$ is a finite subgroup of $\mathbb{T}$ and in this case there is an $n \in \mathbb{N}$ such that Range $(\Theta)=\left\{\exp \left(\frac{2 \pi i p}{n}\right) \mid p \in \mathbb{Z}\right\} \cong \mathbb{Z}_{n}$, which we will call the order of $\Theta$. Throughout this note we will assume that $\Theta$ is rational.

By a result of Brenken [7], if the order of $\Theta$ is non-trivial, that is, larger than one, we can assume that $\mathcal{A}_{\Theta}$ is isomorphic to the following crossed product,

$$
A_{1 / q_{1}} \otimes \cdots \otimes A_{1 / q_{\ell-1}} \otimes A_{r / q_{\ell}} \otimes C\left(\mathbb{T}^{s}\right)
$$

where $r, q_{j}, j=1, \ldots, \ell$, are positive integers, $s=d-2 \ell$, and $\left(r, q_{\ell}\right)=1,1<$ $q_{\ell}|\ldots| q_{1}, q_{1}=$ order of $\Theta$. For ease of notation in what follows we will denote by $\mathcal{A}_{\Theta}^{\prime}$ the tensor product $A_{1 / q_{1}} \otimes \cdots \otimes A_{1 / q_{\ell-1}} \otimes A_{r / q_{\ell}}$. With this decomposition we can obviously generalize the action $\pi$ on $A_{\theta}$ to an action of $S L(2, \mathbb{Z})$ on $\mathcal{A}_{\Theta}$. Here we wish to consider only the diagonal situation by letting $\pi$ act on each non-commutative component of the crossed product and have no action on the commutative component. That is, we will define $\Pi: S L(2, \mathbb{Z}) \rightarrow \operatorname{Aut}\left(\mathcal{A}_{\Theta}\right)$ by

$$
\Pi=\pi \otimes \cdots \otimes \pi \otimes i d .
$$

We will generalize the results for the rotation algebra to this action $\Pi$ on rational non-commutative tori. This is, of course, a restriction since there are many ways to generalize $\pi$, including different actions on different components. The automorphism group, in this case, is larger than that considered. Note, however, that 
in general an action of $S L(d, \mathbb{Z}), d>2$, is not possible and for general higherdimensional tori neither is the action described above unless we restrict to tensor products of rotation algebras. Both of these questions, linked with the structure of the automorphism group in general, require further study.

More precisely, building on those for the rotation algebra we will give a description of $\mathcal{A}_{\Theta}^{\mathbb{Z}_{i}}, i=2,3,4,6$, as Morita equivalent to trivial matrix bundle $C^{*}$-algebras over a certain quotient space with exceptional fibers at a certain number of fixed points and hence deduce their $K$-theory. To save having a large list of special cases, similar to that for small values of $q$ in the rotation algebra case, we will suppose $q_{j}$ is larger than the order of the considered automorphism for all $j=1, \ldots, \ell$.

\section{An alternative Description for the algebra $\mathcal{A}_{\Theta}$}

Firstly recall the following explicit description of the rotation algebra $A_{\frac{p}{q}},(p, q)=$ 1 , see for example $[15,4,11,12,13]$.

$$
A_{\frac{p}{q}} \cong\left\{\begin{array}{l|l}
f \in C(\mathbb{R} \times \mathbb{R}) \otimes M_{q} & \begin{array}{c}
f(x, y+n)=\left(\alpha_{1, q}\right)^{n} f(x, y), \quad 0 \leq x \leq 1, \\
f(x+m, y)=\left(\alpha_{2, q}\right)^{m} f(x, y), \quad 0 \leq y \leq 1,
\end{array}
\end{array}\right\}
$$

where $\alpha_{1, q}$ and $\alpha_{2, q}$ are the automorphisms of $M_{q}$ defined by

$$
\begin{array}{ll}
\alpha_{1, q}\left(U_{o, q}\right)=U_{o, q}, & \alpha_{1, q}\left(V_{o, q}\right)=\omega_{q} V_{o, q}, \\
\alpha_{2, q}\left(U_{o, q}\right)=\omega_{q} U_{o, q}, & \alpha_{2, q}\left(V_{o, q}\right)=V_{o, q},
\end{array}
$$

with $\omega_{q}=e^{\frac{2 \pi i}{q}}, \rho_{q}=\omega_{q}^{p}, U_{o, q}=\left(\delta_{k}^{t} \rho_{q}^{t}\right)_{k, t=0, \ldots, q-1}$ and $V_{o, q}=\left(\delta_{k}^{t-1(\bmod q)}\right)_{k, t=0, \ldots, q-1}$.

Note that $U_{o, q}$ and $V_{o, q}$ generate $M_{q}$ and the two unitary generators $u_{1}$ and $u_{2}$ of $A_{\frac{p}{q}}$ in this description correspond respectively to the functions,

$$
U_{q}(x, y)=\omega_{q}^{x} U_{o, q}, \quad \text { and } \quad V_{q}(x, y)=\omega_{q}^{y} V_{o, q}
$$

Hence, since the elements of type $f_{1}\left(x_{1}, y_{1}\right) \otimes \cdots \otimes f_{\ell}\left(x_{\ell}, y_{\ell}\right), f_{j} \in A_{1 / q_{j}}, j=$ $1, \ldots, \ell-1, f_{\ell} \in A_{r / q_{\ell}}$ generate $\mathcal{A}_{\Theta}^{\prime}$, we get that

$$
\mathcal{A}_{\Theta} \cong\left\{\begin{array}{c|c}
f \in C\left((\mathbb{R} \times \mathbb{R})^{\ell}\right) & \begin{array}{c}
f\left(x_{1}, y_{1}, \ldots, x_{j}, y_{j}+n, \ldots, x_{\ell}, y_{\ell}, t\right) \\
=\left({ }^{j} \tilde{\alpha}_{1, q_{j}}\right)^{n} f\left(x_{1}, y_{1}, \ldots, x_{j}, y_{j}, \ldots, x_{\ell}, y_{\ell}, t\right), \\
f\left(x_{1}, y_{1}, \ldots, x_{j}+m, y_{j}, \ldots, x_{\ell}, y_{\ell}, t\right) \\
=M_{Q} \otimes C\left(\mathbb{T}^{j} \tilde{\alpha}_{2, q_{j}}\right)^{m} f\left(x_{1}, y_{1}, \ldots, x_{j}, y_{j}, \ldots, x_{\ell}, y_{\ell}, t\right), \\
0 \leq x_{j}, y_{j} \leq 1, j=1, \ldots, \ell
\end{array}
\end{array}\right\}
$$

where $Q=\prod_{j=1}^{\ell} q_{j}$ and ${ }^{j} \tilde{\alpha}_{k, q_{j}}=1 \otimes \cdots \otimes \underbrace{\alpha_{k, q_{j}}}_{j \text {-th slot }} \otimes 1 \otimes \cdots \otimes 1$ is an automorphism of $M_{Q}$ for $k=1,2$ and $j=1, \ldots, \ell$.

The $2 \ell$ unitary generators of $\mathcal{A}_{\Theta}^{\prime}$ in the above description correspond to the functions

$$
\begin{aligned}
& U_{q_{j}}=1 \otimes \cdots \otimes 1 \otimes \underbrace{\omega_{q_{j}}^{x_{j}} U_{o, q_{j}}}_{j \text {-th slot }} \otimes 1 \otimes \cdots \otimes 1, \quad j=1, \ldots, \ell, \\
& V_{q_{j}}=1 \otimes \cdots \otimes 1 \otimes \underbrace{\omega_{q_{j}}^{y_{j}} V_{o, q_{j}}}_{j \text {-th slot }} \otimes 1 \otimes \cdots \otimes 1, \quad j=1, \ldots, \ell .
\end{aligned}
$$




\section{The fixed point subalgebra $\mathcal{A}_{\Theta}^{S}$}

The generator $\left(\begin{array}{cc}-1 & 0 \\ 0 & -1\end{array}\right)$ of the subgroup $\mathbb{Z}_{2}$ of $S L(2, \mathbb{Z})$ corresponds to the automorphism $S$ of $\mathcal{A}_{\Theta}$ given by

$$
(S f)\left(x_{1}, y_{1}, \ldots, x_{\ell}, y_{\ell}, t\right)=S_{0}\left(f\left(1-x_{1}, 1-y_{1}, \ldots, 1-x_{\ell}, 1-y_{\ell}, t\right)\right),
$$

where $S_{0}=\sigma_{o, q_{1}} \otimes \cdots \otimes \sigma_{o, q_{\ell}}$ and $\sigma_{o, q}, q \in \mathbb{N}$, is the automorphism of $M_{q}$ determined by,

$$
\sigma_{o, q}\left(U_{o, q}\right)=\omega_{q}^{*} U_{o, q}^{*}, \quad \sigma_{o, q}\left(V_{o, q}\right)=\omega_{q}^{*} V_{o, q}^{*}
$$

(It is in fact an easy computation to check this.) For $d=2, \ell=1, s=0$ the above expression for $S$ was introduced in [4] and in this more general case the analysis will follow a similar procedure to that paper. It is also clear that $S$ also induces an automorphism, which we will still call $S$, of $\mathcal{A}_{\Theta}^{\prime}$, since it acts as the identity on $C\left(\mathbb{T}^{s}\right)$. Moreover the fixed point algebra $\mathcal{A}_{\Theta}^{S}$ is isomorphic to $\mathcal{A}_{\Theta}^{\prime S} \otimes C\left(\mathbb{T}^{s}\right)$. We will therefore proceed to describe $\mathcal{A}_{\Theta}^{\prime S}$.

To describe the fixed point algebra $\mathcal{A}_{\Theta}^{\prime S}$ the idea is to make use of the splitting of $S$ into an automorphism of $M_{Q}$ and a homeomorphism of the underlying space introduced above to characterize $\mathcal{A}_{\Theta}^{\prime S}$ as a $C^{*}$-matrix bundle over the quotient space, which is an orbifold. More precisely, let $\sigma$ be the homeomorphism of $(\mathbb{R} \times \mathbb{R})^{\ell}$ given by

$$
\left(x_{1}, y_{1}, \ldots, x_{\ell}, y_{\ell}\right) \stackrel{\sigma}{\mapsto}\left(1-x_{1}, 1-y_{1}, \ldots, 1-x_{\ell}, 1-y_{\ell}\right) .
$$

Note that $\sigma$ also induces an order two homeomorphism of $\mathbb{T}^{2 \ell}$, which we will still call $\sigma$. Moreover,

$$
\mathbb{T}^{2 \ell} / \sigma=\left\{\begin{array}{c}
\left(x_{1}, y_{1}, \ldots, x_{\ell}, y_{\ell}\right) \\
\in([0,1] \times[0,1])^{\ell}
\end{array} \mid y_{1} \leq x_{1} \text { with the equivalences below }\right\},
$$

for $x_{1}=1:\left(1, y_{1}, x_{2}, y_{2}, \ldots, x_{\ell}, y_{\ell}\right) \sim\left(1,1-y_{1}, \ldots, 1-x_{\ell}, 1-y_{\ell}\right)$, for $y_{1}=0:\left(x_{1}, 0, x_{2}, y_{2}, \ldots, x_{\ell}, y_{\ell}\right) \sim\left(1-x_{1}, 0, \ldots, 1-x_{\ell}, 1-y_{\ell}\right)$, for $y_{1}=x_{1}$ : $\left(x_{1}, x_{1}, x_{2}, y_{2}, \ldots, x_{\ell}, y_{\ell}\right) \sim\left(1-x_{1}, 1-x_{1}, \ldots, 1-x_{\ell}, 1-y_{\ell}\right)$, and for $i=1, \ldots, \ell$ :

$$
\begin{aligned}
\left(x_{1}, y_{1}, \ldots, 1, y_{i}, \ldots, x_{\ell}, y_{\ell}\right) & \sim\left(x_{1}, y_{1}, \ldots, 0, y_{i}, \ldots, x_{\ell}, y_{\ell}\right), \\
\left(x_{1}, y_{1}, \ldots, x_{i}, 1, \ldots, x_{\ell}, y_{\ell}\right) & \sim\left(x_{1}, y_{1}, \ldots, x_{i}, 0, \ldots, x_{\ell}, y_{\ell}\right) .
\end{aligned}
$$

Hence the fixed point algebra $\mathcal{A}_{\Theta}^{\prime S}$ is isomorphic to

$$
\mathcal{A}_{\Theta}^{\prime S} \cong\left\{f \in C\left(([0,1] \times[0,1])^{\ell} \otimes M_{Q} \text { with } y_{1} \leq x_{1}\right) \text { with the conditions below }\right\} \text {. }
$$

For $x_{1}=1: f\left(1, y_{1}, x_{2}, y_{2}, \ldots, x_{\ell}, y_{\ell}\right)={ }^{1} \tilde{\alpha}_{2, q_{1}}^{-1} S_{0} f\left(1,1-y_{1}, \ldots, 1-x_{\ell}, 1-y_{\ell}\right)$, for $y_{1}=0: f\left(x_{1}, 0, x_{2}, y_{2}, \ldots, x_{\ell}, y_{\ell}\right)={ }^{1} \tilde{\alpha}_{1, q_{1}} S_{0} f\left(1-x_{1}, 0, \ldots, 1-x_{\ell}, 1-y_{\ell}\right)$, for $y_{1}=x_{1}: f\left(x_{1}, x_{1}, x_{2}, y_{2}, \ldots, x_{\ell}, y_{\ell}\right)=S_{0} f\left(1-x_{1}, 1-x_{1}, \ldots, 1-x_{\ell}, 1-y_{\ell}\right)$, and for $i=1, \ldots, \ell$ :

$$
\begin{aligned}
f\left(x_{1}, y_{1}, \ldots, 1, y_{i}, \ldots, x_{\ell}, y_{\ell}\right) & ={ }^{i} \tilde{\alpha}_{2, q_{i}} f\left(x_{1}, y_{1}, \ldots, 0, y_{i}, \ldots, x_{\ell}, y_{\ell}\right), \\
f\left(x_{1}, y_{1}, \ldots, x_{i}, 1, \ldots, x_{\ell}, y_{\ell}\right) & ={ }^{i} \tilde{\alpha}_{1, q_{i}} f\left(x_{1}, y_{1}, \ldots, x_{i}, 0, \ldots, x_{\ell}, y_{\ell}\right) .
\end{aligned}
$$


With analogous computations to the case of rotation algebras (cf. $[4,11,12,13]$ ) the fixed point subalgebra is shown to be Morita equivalent to

$\mathcal{A}_{\Theta}^{S} \sim\left\{f \in C\left(\mathbb{T}^{2 \ell} / \sigma\right) \otimes M_{Q} \mid f\left(\alpha_{j}\right)\right.$ is 2-block diagonal, $\left.j=1, \ldots, 2^{2 \ell}\right\} \otimes C\left(\mathbb{T}^{s}\right)$, where $\alpha_{j}, j=1, \ldots, 2^{2 \ell}$, are the (order 2) elliptic points of $\mathbb{T}^{2 \ell} / \sigma$, that is, the images of the (order 2) fixed points of the automorphism $\sigma$ on $\mathbb{T}^{2 \ell}$. Namely, $\alpha_{j}=\left[A_{j_{1}}, \ldots, A_{j_{\ell}}\right]$ with $A_{j_{k}} \in\{(0,0),(1 / 2,1 / 2),(1 / 2,0),(1,1 / 2)\}$ ([ ] denotes the equivalence class of the given point in the quotient orbifold). The fact that these are the only elliptic points of $\mathbb{T}^{2 \ell} / \sigma$ follows from analogous facts as those for dimension two.

\section{The fiXed point subalgebra $\mathcal{A}_{\Theta}^{T}$}

The generator $\left(\begin{array}{cc}0 & 1 \\ -1 & 0\end{array}\right)$ of the subgroup $\mathbb{Z}_{4}$ of $S L(2, \mathbb{Z})$ corresponds to the automorphism $T$ of $\mathcal{A}_{\Theta}$ given by

$$
(T f)\left(x_{1}, y_{1}, \ldots, x_{\ell}, y_{\ell}, t\right)=T_{0}\left(f\left(1-y_{1}, x_{1}, \ldots, 1-y_{\ell}, x_{\ell}, t\right)\right),
$$

where $T_{0}=\tau_{o, q_{1}} \otimes \cdots \otimes \tau_{o, q_{\ell}}$ and $\tau_{o, q}$ is the automorphism of $M_{q}$ determined by

$$
\tau_{o, q}\left(U_{o, q}\right)=\omega_{q}^{*} V_{o, q}^{*}, \quad \tau_{o, q}\left(V_{o, q}\right)=U_{o, q}
$$

As in the previous case we use this splitting of $T$ to characterize $\mathcal{A}_{\Theta}^{T}$ as a $C^{*}$-matrix bundle over the quotient space, which is an orbifold. More precisely, let $\tau$ be the homeomorphism of $(\mathbb{R} \times \mathbb{R})^{\ell}$ given by

$$
\left(x_{1}, y_{1}, \ldots, x_{\ell}, y_{\ell}\right) \stackrel{\tau}{\mapsto}\left(1-y_{1}, x_{1}, 1-y_{2}, x_{2}, \ldots, 1-y_{\ell}, x_{\ell}\right) .
$$

This also induces a homeomorphism of $\mathbb{T}^{2 \ell}$, which we will still call $\tau$ and note that $\tau^{2}=\sigma$. Now,

$\mathbb{T}^{2 \ell} / \tau=\left\{\begin{array}{c}\left(x_{1}, y_{1}, \ldots, x_{\ell}, y_{\ell}\right) \\ \in([0,1] \times[0,1])^{\ell}\end{array} \mid y_{1} \leq \min \left\{x_{1}, 1-x_{1}\right\}\right.$ with the equivalences below $\}$,

for $y_{1}=0:\left(x_{1}, 0, x_{2}, y_{2}, \ldots, x_{\ell}, y_{\ell}\right) \sim\left(1-x_{1}, 0,1-x_{2}, 1-y_{2}, \ldots, 1-x_{\ell}, 1-y_{\ell}\right)$, for $y_{1}=x_{1} \leq 1 / 2:\left(x_{1}, x_{1}, x_{2}, y_{2}, \ldots, x_{\ell}, y_{\ell}\right) \sim\left(1-x_{1}, x_{1}, 1-y_{2}, x_{2}, \ldots, 1-y_{\ell}, x_{\ell}\right)$, and for $i=1, \ldots, \ell$ :

$$
\begin{aligned}
\left(x_{1}, y_{1}, \ldots, 1, y_{i}, \ldots, x_{\ell}, y_{\ell}\right) & \sim\left(x_{1}, y_{1}, \ldots, 0, y_{i}, \ldots, x_{\ell}, y_{\ell}\right), \\
\left(x_{1}, y_{1}, \ldots, x_{i}, 1, \ldots, x_{\ell}, y_{\ell}\right) & \sim\left(x_{1}, y_{1}, \ldots, x_{i}, 0, \ldots, x_{\ell}, y_{\ell}\right) .
\end{aligned}
$$

Hence the fixed point algebra $\mathcal{A}_{\Theta}^{\prime T}$ is isomorphic to

$\mathcal{A}_{\Theta}^{\prime T} \cong\left\{f \in C\left(([0,1] \times[0,1])^{\ell} \otimes M_{Q}, y_{1} \leq \min \left\{x_{1}, 1-x_{1}\right\}\right):\right.$ the conditions below $\}$.

For $y_{1}=0: f\left(x_{1}, 0, x_{2}, y_{2}, \ldots, x_{\ell}, y_{\ell}\right)={ }^{1} \tilde{\alpha}_{1, q} S_{0} f\left(1-x_{1}, 0, \ldots, 1-x_{\ell}, 1-y_{\ell}\right)$, for $y_{1}=x_{1} \leq 1 / 2: f\left(x_{1}, x_{1}, x_{2}, y_{2}, \ldots, x_{\ell}, y_{\ell}\right)=T_{0} f\left(1-x_{1}, x_{1}, \ldots, 1-y_{\ell}, x_{\ell}\right)$, and for $i=1, \ldots, \ell$ :

$$
\begin{aligned}
& f\left(x_{1}, y_{1}, \ldots, 1, y_{i}, \ldots, x_{\ell}, y_{\ell}\right)={ }^{i} \tilde{\alpha}_{2, q_{i}} f\left(x_{1}, y_{1}, \ldots, 0, y_{i}, \ldots, x_{\ell}, y_{\ell}\right), \\
& f\left(x_{1}, y_{1}, \ldots, x_{i}, 1, \ldots, x_{\ell}, y_{\ell}\right)={ }^{i} \tilde{\alpha}_{1, q_{i}} f\left(x_{1}, y_{1}, \ldots, x_{i}, 0, \ldots, x_{\ell}, y_{\ell}\right),
\end{aligned}
$$

The elliptic points of $\mathbb{T}^{2 \ell} / \tau$ are of the following types: 
Order 4. $\alpha_{j}=\left[A_{j_{1}}, \ldots, A_{j_{\ell}}\right]$ where $A_{j_{k}} \in\{(1 / 2,1 / 2),(0,0)\}$. There are $2^{\ell}$ distinct such points in $\mathbb{T}^{2 \ell} / \tau$.

Order 2. $\beta_{j}=\left[B_{j_{1}}, \ldots, B_{j_{\ell}}\right]$ where $B_{j_{k}} \in\{(1 / 2,1 / 2),(0,0),(1,1 / 2),(1 / 2,0)\}$ with at least one of the $B_{j_{k}}$ in each $\beta_{j}$ different from $(1 / 2,1 / 2)$ and $(0,0)$ (i.e. each $\beta_{j}$ is not an $\left.\alpha_{j}\right)$. There are $\left(2^{2 \ell}-2^{\ell}\right) / 2$ distinct such points in the quotient orbifold $\mathbb{T}^{2 \ell} / \tau$.

With analogous computations to the case of rotation algebras the fixed point subalgebra is shown to be Morita equivalent to

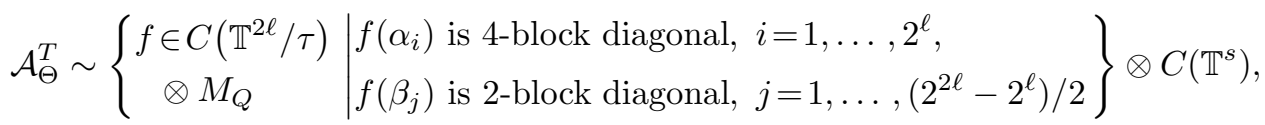

where $\alpha_{i}, i=1, \ldots, 2^{\ell}$, are the order 4 elliptic points of $\mathbb{T}^{2 \ell} / \tau$ and $\beta_{j}, j=$ $1, \ldots,\left(2^{2 \ell}-2^{\ell}\right) / 2$, are the order two elliptic points.

\section{The fixed point subalgebra $\mathcal{A}_{\Theta}^{Z}$}

The generator $\left(\begin{array}{cc}-1 & -1 \\ 1 & 0\end{array}\right)$ of the subgroup $\mathbb{Z}_{3}$ of $S L(2, \mathbb{Z})$ corresponds to the automorphism $Z$ of $\mathcal{A}_{\Theta}$ given by

$$
(Z f)\left(x_{1}, y_{1}, \cdots, x_{\ell}, y_{\ell}, t\right)=Z_{0}\left(f\left(\delta_{1}-x_{1}+y_{1},-x_{1}, \cdots, \delta_{\ell}-x_{\ell}+y_{\ell},-x_{\ell}, t\right)\right),
$$

where $Z_{0}=\zeta_{o, q_{1}} \otimes \cdots \otimes \zeta_{o, q_{\ell}}$ and $\zeta_{o, q_{j}}$ is the automorphism of $M_{q_{j}}$ determined by

$$
\zeta_{o, q_{j}}\left(U_{o, q_{j}}\right)=e^{\pi i\left[1-\delta_{j}\right] \epsilon_{j} /\left(2 q_{j}\right)} U_{o, q_{j}}^{*} V_{o, q_{j}}, \quad \zeta_{o, q_{j}}\left(V_{o, q_{j}}\right)=U_{o, q_{j}}^{*},
$$

where $\delta_{j}=\left[-1+(-1)^{q_{j}}\right] \epsilon_{j} / 4$, with $\epsilon_{j}=1$ for $j=1, \ldots, \ell-1$ and $\epsilon_{\ell}=r$. Let $\zeta$ denote the homeomorphism of $\mathbb{T}^{2 \ell}$ induced by the transformation of $(\mathbb{R} \times \mathbb{R})^{\ell}$ given by

$$
\left(x_{1}, y_{1}, \ldots, x_{\ell}, y_{\ell}\right) \stackrel{\zeta}{\mapsto}\left(\delta_{1}-x_{1}+y_{1},-x_{1}, \ldots, \delta_{\ell}-x_{\ell}+y_{\ell},-x_{\ell}\right) .
$$

A domain for $\zeta$, similar to $\sigma$ and $\tau$, can be easily computed, but its description is rather lengthy so it will not be given. The (order 3 ) elliptic points of the orbifold $\mathbb{T}^{2 \ell} / \zeta$ are given by $\alpha_{j}=\left[A_{j_{1}}, \ldots, A_{j_{\ell}}\right]$ where for $k=1, \ldots, \ell-1, A_{j_{k}} \in\{(0,0)$, $(2 / 3,1 / 3),(1 / 3,2 / 3)\}$ if $q_{k}$ even or $A_{j_{k}} \in\{(-1 / 6,1 / 6),(1 / 2,1 / 2),(1 / 6,5 / 6)\}$ if $q_{k}$ odd, while $A_{j_{\ell}} \in\{(0,0),(2 / 3,1 / 3),(1 / 3,2 / 3)\}$ if either $q_{\ell}$ or $r$ is even or $A_{j_{k}} \in\{(-1 / 6,1 / 6),(1 / 2,1 / 2),(1 / 6,5 / 6)\}$ if $q_{\ell}$ and $r$ are both odd. There are $3^{\ell}$ elliptic points in $\mathbb{T}^{2 \ell} / \zeta$.

Hence $\mathcal{A}_{\Theta}^{Z}$ is Morita equivalent to

$$
\mathcal{A}_{\Theta}^{Z} \sim\left\{f \in C\left(\mathbb{T}^{2 \ell} / \zeta\right) \otimes M_{Q} \mid f\left(\alpha_{i}\right) \text { is 3-block diagonal, } i=1, \ldots, 3^{\ell}\right\} \otimes C\left(\mathbb{T}^{s}\right)
$$

where $\alpha_{i}, i=1, \ldots, 3^{\ell}$, are the order 3 elliptic points of $\mathbb{T}^{2 \ell} / \zeta$.

$$
\text { 6. The Fixed point subalgebra } \mathcal{A}_{\Theta}^{E}
$$

The generator $\left(\begin{array}{cc}0 & -1 \\ 1 & 1\end{array}\right)$ of the subgroup $\mathbb{Z}_{6}$ of $S L(2, \mathbb{Z})$ corresponds to the automorphism $E$ of $\mathcal{A}_{\Theta}$ given by

$$
(E f)\left(x_{1}, y_{1}, \ldots, x_{\ell}, y_{\ell}, t\right)=E_{0}\left(f\left(y_{1}, y_{1}-x_{1}+\delta_{1}, \ldots, y_{\ell}, y_{\ell}-x_{\ell}+\delta_{\ell}, t\right)\right),
$$


where $E_{0}=\eta_{o, q_{1}} \otimes \cdots \otimes \eta_{o, q_{\ell}}$ and $\eta_{o, q_{j}}$ is the automorphism of $M_{q_{j}}$ determined by

$$
\eta_{o, q_{j}}\left(U_{o, q_{j}}\right)=V_{o, q_{j}}, \quad \eta_{o, q_{j}}\left(V_{o, q_{j}}\right)=e^{\pi i\left[1-\delta_{j}\right] \epsilon_{j} /\left(2 q_{j}\right)} U_{o, q_{j}}^{*} V_{o, q_{j}}
$$

Let $\eta$ denote the homeomorphism of $\mathbb{T}^{2 \ell}$ induced by the transformation of $(\mathbb{R} \times \mathbb{R})^{\ell}$ given by

$$
\left(x_{1}, y_{1}, \ldots, x_{\ell}, y_{\ell}\right) \stackrel{\eta}{\mapsto}\left(y_{1}, y_{1}-x_{1}+\delta_{1}, \ldots, y_{\ell}, y_{\ell}-x_{\ell}+\delta_{\ell}\right)
$$

The elliptic points of $\mathbb{T}^{2 \ell} / \eta$ are as follows:

Order 6. $\alpha=\left[A_{1}, \ldots, A_{\ell}\right]$ where for $k=1, \ldots, \ell-1, A_{k}=(0,0)$ if $q_{k}$ even or $A_{k}=(1 / 2,1 / 2)$ if $q_{k}$ odd, while $A_{\ell}=(0,0)$ if either $q_{\ell}$ or $r$ is even and $A_{\ell}=$ $(1 / 2,1 / 2)$ if $q_{\ell}$ and $r$ are both odd.

Order 3. $\beta_{j}=\left[B_{j_{1}}, \ldots, B_{j_{\ell}}\right]$ where for $k=1, \ldots, \ell-1, B_{j_{k}} \in\{(0,0),(1 / 3,2 / 3)$, $(2 / 3,1 / 3)\}$ if $q_{k}$ even, or $B_{j_{k}} \in\{(1 / 2,1 / 2),(1 / 6,5 / 6),(-1 / 6,1 / 6)\}$ if $q_{k}$ odd, while $B_{j_{\ell}} \in\{(0,0),(1 / 3,2 / 3),(2 / 3,1 / 3)\}$ if either $q_{\ell}$ or $r$ is even and $B_{j_{\ell}} \in\{(1 / 2,1 / 2)$, $(1 / 6,5 / 6),(-1 / 6,1 / 6)\}$ if $q_{\ell}$ and $r$ are both odd. Also at least one of the $B_{j_{k}}$ in each $\beta_{j}$ is different from $(1 / 2,1 / 2)$ and $(0,0)$ (i.e. each $\beta_{j}$ is not $\alpha$ ).

Order 2. $\gamma_{j}=\left[C_{j_{1}}, \ldots, C_{j_{\ell}}\right]$ where $C_{j_{k}} \in\{(1 / 2,1 / 2),(0,0),(1,1 / 2),(1 / 2,0)\}$ with at least one of the $C_{j_{k}}$ in each $\gamma_{j}$ different from $(1 / 2,1 / 2)$ and $(0,0)$ (i.e. each $\gamma_{j}$ is not $\alpha$ ).

In the quotient orbifold $\mathbb{T}^{2 \ell} / \eta$ there is just one order 6 elliptic point, while there are $\left(3^{\ell}-1\right) / 2$ order 3 elliptic points and $\left(4^{\ell}-1\right) / 3$ order 2 ones. Hence $\mathcal{A}_{\Theta}^{E}$ is Morita equivalent to

$\mathcal{A}_{\Theta}^{E} \sim\left\{\begin{array}{c|l}f \in C\left(\mathbb{T}^{2 \ell} / \eta\right) & \begin{array}{l}f(\alpha) \text { is 6-block diagonal, } \\ f\left(\beta_{i}\right) \text { is 3-block diagonal, } i=1, \ldots,\left(3^{\ell}-1\right) / 2, \\ \otimes M_{Q}\end{array} \\ f\left(\gamma_{j}\right) \text { is 2-block diagonal, } j=1, \ldots,\left(4^{\ell}-1\right) / 3\end{array}\right\} \otimes C\left(\mathbb{T}^{s}\right)$,

where $\alpha$ is the order 6 elliptic point, $\beta_{i}, i=1, \ldots\left(3^{\ell}-1\right) / 2$, are the order 3 elliptic points, and $\gamma_{j}, j=1, \ldots,\left(4^{\ell}-1\right) / 3$, are the order 2 elliptic points.

\section{K-THEORY}

In this section we would like to compute the $K$-theory for these fixed point subalgebras, that is,

$$
K_{j}\left(\mathcal{A}_{\Theta}^{X}\right), \quad j=0,1, \quad X=S, T, Z, E .
$$

Since all of these algebras are described in terms of the tensor product of a matrix bundle $C^{*}$-algebra with $C\left(\mathbb{T}^{s}\right)$ the computation reduces to the computation of the K-theory of this matrix bundle $C^{*}$-algebra and an application of Künneth's theorem. To facilitate the description of the necessary computations we will make the following definitions. Let

$$
\begin{gathered}
\mathcal{A}_{0}^{S}=\left\{f \in C\left(\mathbb{T}^{2 \ell} / \sigma\right) \otimes M_{Q} \mid f\left(\alpha_{i}\right) \text { is 2-block diagonal, } i=1, \ldots, 2^{2 \ell}\right\}, \\
\mathcal{A}_{0}^{T}=\left\{f \in C\left(\mathbb{T}^{2 \ell} / \tau\right) \otimes M_{Q} \mid \begin{array}{l}
f\left(\alpha_{i}\right) \text { is 4-block diagonal, } i=1, \ldots, 2^{\ell}, \\
f\left(\beta_{j}\right) \text { is 2-block diagonal, } j=1, \ldots,\left(2^{2 \ell}-2^{\ell}\right) / 2
\end{array}\right\}, \\
\mathcal{A}_{0}^{Z}=\left\{f \in C\left(\mathbb{T}^{2 \ell} / \zeta\right) \otimes M_{Q} \mid f\left(\alpha_{i}\right) \text { is 3-block diagonal, } i=1, \ldots, 3^{\ell}\right\}, \text { and }
\end{gathered}
$$




$$
\mathcal{A}_{0}^{E}=\left\{\begin{array}{l|l}
f \in C\left(\mathbb{T}^{2 \ell} / \eta\right) \otimes M_{Q} & \begin{array}{l}
f(\alpha) \text { is 6-block diagonal, } \\
f\left(\beta_{i}\right) \text { is 3-block diagonal, } i=1, \ldots,\left(3^{\ell}-1\right) / 2, \\
f\left(\gamma_{j}\right) \text { is 2-block diagonal, } j=1, \ldots,\left(4^{\ell}-1\right) / 3
\end{array}
\end{array}\right\} .
$$

Note that $\mathcal{A}_{0}^{X}$ is Morita equivalent to the $C^{*}$-algebra $\mathcal{A}_{\Theta}^{\prime X}, X=S, T, Z$ and $E$ which we introduced above. We will consider the computation in detail for $\mathcal{A}_{0}^{S}$ only, the other three cases requiring analogous calculations. In $\mathcal{A}_{0}^{S}$ we can assume that all the singular points $\alpha_{i}, i=1, \ldots, 2^{2 \ell}$, are inside a small open ball $D$. The $C^{*}$-algebra $\mathcal{D}=\left\{f \in C_{0}(D) \otimes M_{Q} \mid f\left(\alpha_{i}\right)\right.$ is 2-block diagonal, $\left.i=1, \ldots, 2^{2 \ell}\right\}$ is an ideal of $\mathcal{A}_{0}^{S}$ and the standard six-term exact sequence of K-theory associated to it is given below.

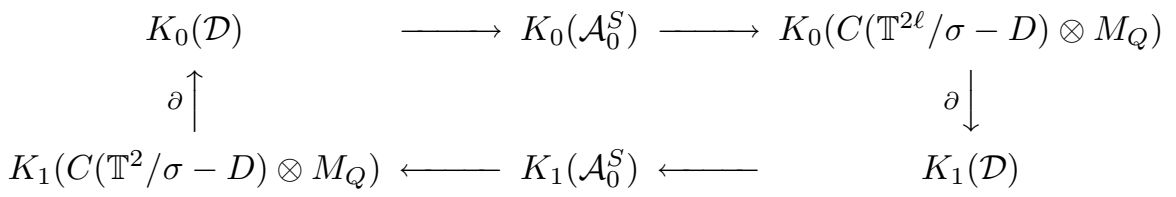

It is easily seen that $K_{0}(\mathcal{D}) \cong \mathbb{Z}^{2^{2 \ell}+1}$ and $K_{1}(\mathcal{D})=0$, so the sequence may be written as follows.

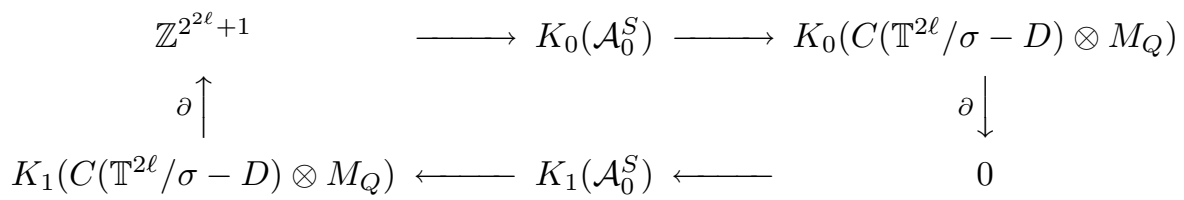

Now note that $\mathbb{T}^{2 \ell} / \sigma$ is an orbifold having as singular set the elliptic points $\alpha_{i}, i=$ $1, \ldots, 2^{2 \ell}$. (This can be seen by solving for the fixed points of $\sigma$.) A neighborhood of $\alpha_{i}$ can be described as $\mathbb{R}^{2 \ell} / \mathbb{Z}_{2}$ and hence it can be given a manifold structure since it is homeomorphic to $\mathbb{R}^{2 \ell}$ itself. It follows that $\mathbb{T}^{2 \ell} / \sigma$ can also be given a manifold structure. Next note that the map $\mathbb{Z}^{2^{2 \ell}+1} \rightarrow K_{0}\left(\mathcal{A}_{0}^{S}\right)$ is injective since the $K$-theory class corresponding to the fundamental cocycle maps to $\left(\begin{array}{cccc}1 & 0 & \ldots & 0 \\ 0 & 0 & \ldots & 0 \\ \vdots & \vdots & \ddots & \vdots \\ 0 & 0 & \ldots & 0\end{array}\right)$ in $K_{0}\left(\mathcal{A}_{0}^{S}\right)$ and each of the other generators correspond to block-diagonal projections of type $\left(\begin{array}{cccc}0 & \ldots & 0 & 0 \\ \vdots & \ddots & \ddots & \vdots \\ 0 & \vdots & \vdots \\ 0 & \cdots & 0 & 0\end{array}\right)$ at $\alpha_{i}$ whose image still give rise to generators in $K_{0}\left(\mathcal{A}_{0}^{S}\right)$.

Hence it follows that

$$
K_{0}\left(\mathcal{A}_{0}^{S}\right) \cong \mathbb{Z}^{2^{2 \ell}} \oplus K^{0}\left(\mathbb{T}^{2 \ell} / \sigma\right), \quad K_{1}\left(\mathcal{A}_{0}^{S}\right) \cong K^{1}\left(\mathbb{T}^{2 \ell} / \sigma\right) .
$$

Analogously, for the other three cases, one can prove,

$$
\begin{aligned}
& K_{0}\left(\mathcal{A}_{0}^{T}\right) \cong \mathbb{Z}^{\left(2^{2 \ell}-2^{\ell}\right) / 2} \oplus \mathbb{Z}^{3 \cdot 2^{\ell}} \oplus K^{0}\left(\mathbb{T}^{2 \ell} / \tau\right) \\
& K_{0}\left(\mathcal{A}_{0}^{Z}\right) \cong \mathbb{Z}^{2 \cdot 3^{\ell}} \oplus K^{0}\left(\mathbb{T}^{2 \ell} / \zeta\right), \\
& K_{0}\left(\mathcal{A}_{0}^{E}\right) \cong \mathbb{Z}^{\left(4^{\ell}-1\right) / 3} \oplus \mathbb{Z}^{2 \cdot\left(3^{\ell}-1\right) / 2} \oplus \mathbb{Z}^{5} \oplus K^{0}\left(\mathbb{T}^{2 \ell} / \eta\right), \\
& K_{1}\left(\mathcal{A}_{0}^{X}\right) \cong K^{1}\left(\mathbb{T}^{2 \ell} / \alpha\right), \text { where } X=T, Z, E \text { and } \alpha=\tau, \zeta, \eta \text { respectively. }
\end{aligned}
$$


Note also that $K^{0}\left(\mathbb{T}^{2 \ell} / \sigma\right)=H^{e v}\left(\mathbb{T}^{2 \ell}\right)^{\sigma} \cong \mathbb{Z}^{2 \ell-1}$ and $K^{1}\left(\mathbb{T}^{2 \ell} / \sigma\right)=H^{o d}\left(\mathbb{T}^{2 \ell}\right)^{\sigma}=0$. Moreover $K^{0}\left(\mathbb{T}^{2 \ell} / \alpha\right)=H^{e v}\left(\mathbb{T}^{2 \ell}\right)^{\alpha} \cong \mathbb{Z}^{2^{\ell}}$ and $K^{1}\left(\mathbb{T}^{2 \ell} / \alpha\right)=H^{o d}\left(\mathbb{T}^{2 \ell}\right)^{\alpha}=0$, where $\alpha=\tau, \zeta$ and $\eta$. Hence,

$$
\begin{aligned}
& K_{0}\left(\mathcal{A}_{\Theta}^{S}\right) \cong K_{1}\left(\mathcal{A}_{\Theta}^{S}\right) \cong \mathbb{Z}^{3 \cdot 2^{2 \ell-1}} \otimes \mathbb{Z}^{2^{s-1}} \\
& K_{0}\left(\mathcal{A}_{\Theta}^{T}\right) \cong K_{1}\left(\mathcal{A}_{\Theta}^{T}\right) \cong \mathbb{Z}^{2^{2 \ell-1}+7 \cdot 2^{\ell-1}} \otimes \mathbb{Z}^{2^{s-1}}, \\
& K_{0}\left(\mathcal{A}_{\Theta}^{Z}\right) \cong K_{1}\left(\mathcal{A}_{\Theta}^{Z}\right) \cong \mathbb{Z}^{2 \cdot 3^{\ell}+2^{\ell}} \otimes \mathbb{Z}^{2^{s-1}} \\
& K_{0}\left(\mathcal{A}_{\Theta}^{E}\right) \cong K_{1}\left(\mathcal{A}_{\Theta}^{E}\right) \cong \mathbb{Z}^{3^{\ell}+2^{\ell}+4+\left(\left(4^{\ell}-1\right) / 3\right)} \otimes \mathbb{Z}^{2^{s-1}}
\end{aligned}
$$

Note that the results of the above calculations for $K_{0}\left(\mathcal{A}_{\Theta}^{S}\right)$ and $K_{1}\left(\mathcal{A}_{\Theta}^{S}\right)$ are the same as for $\Theta$ completely irrational [14].

\section{REFERENCES}

1. B. Blackadar, K-Theory for Operator Algebras, MSRI Publications, vol. 5, Springer-Verlag, New York, 1986. MR 88g:46082

2. F. Boca, On the flip fixed point algebra in certain non commutative tori, preprint.

3. O. Bratteli, G.A. Elliott, D.E. Evans and A. Kishimoto, Non commutative spheres I, International J. Math. 2 (1991), 139-166. MR 92d:58008

4. O. Bratteli, G.A. Elliott, D.E. Evans and A. Kishimoto, Non commutative spheres II, Rational Rotations, J. Operator Theory 27 (1992), 53-85. MR 94j:46068

5. O. Bratteli and A. Kishimoto, Non-commutative spheres III. Irrational rotations, Commun. Math. Phys. 147 (1992), 605-624. MR 93g:58008

6. B.A. Brenken, Representations and automorphisms of the irrational rotation algebra, Pacific J. Math. 111 (1984), 257-282. MR 86a:46089

7. B.A. Brenken, A classification of some noncommutative tori, Rocky Mountain J. Math 20 (1990), 389-397. MR 91e:46099

8. G. A. Elliott, On the $K$-theory of the $C^{*}$-algebra generated by a projective representation of a torsion-free discrete abelian group, Operator Algebras and Group Representations, vol. 1, Pitman, London, 1984, pp. 157-184. MR 85m:46067

9. G.A. Elliott and M. Rørdam, The automorphism group of the irrational rotation $C^{*}$-algebra, preprint.

10. C. Farsi and N. Watling, Fixed point subalgebras of the rotation algebra, C.R. Math. Rep. Acad. Sci. Canada XIII (1991), 75-80. MR 92j:46117b

11. C. Farsi and N. Watling, Quartic algebras, Canad. J. Math 44 (1992), 1167-1191. MR 94b: 46085

12. C. Farsi and N. Watling, Elliptic algebras, J. Func. Anal. 118 (1993), 1-21. MR 95h:46099

13. C. Farsi and N. Watling, Cubic algebras, J. Operator Theory 30 (1993), 243-266. MR 96f: 46126

14. C. Farsi and N. Watling, Symmetrized non-commutative tori, Math. Ann. 296 (1993), 739741. MR 94k:46130

15. R. Høegh-Krohn and T. Skjelbred, Classification of $C^{*}$-algebras admitting ergodic actions on the two dimensional torus, J. Reine Angewandte Math. 328 (1981), 1-8. MR 83m:46082

16. A. Kumjian, On the $K$-theory of the symmetrized non-commutative torus, C.R. Math. Rep. Acad. Sci. Canada XII (1990), 87-89. MR 92b:46115

17. Y. Watatani, Toral automorphisms on irrational rotation algebras, Math. Japonica 26 (1981), 479-484. MR 82m:46073

Carla Farsi, Department of Mathematics, University of Colorado, Campus Box 395, BOUlder, COLORADO 80309-0395.

E-mail address: farsi@euclid.colorado.edu

Neil Watling, Department of Mathematics, Widener University, 1 University Place, Chester, Pennsylvania 19013-5792

E-mail address: watling@kuratowski.math.widener.edu 